\title{
Exploring Voltage Mediated Delamination of Suspended 2D Materials as a Cause of Commonly Observed Breakdown
}

DOI:

10.1021/acs.jpcc.9b08500

\section{Document Version}

Accepted author manuscript

Link to publication record in Manchester Research Explorer

\section{Citation for published version (APA):}

Loessberg-zahl, J., De Bruijn, D. S., Van Den Beld, W. T. E., Dollekamp, E., Grady, E., Keerthi, A., Bomer, J. G., Boya, R., Zandvliet, H. J. W., Bol, A. A., Van Den Berg, A., \& Eijkel, J. C. T. (2020). Exploring Voltage Mediated Delamination of Suspended 2D Materials as a Cause of Commonly Observed Breakdown. The Journal of Physical Chemistry C, 124(1), 430-435. https://doi.org/10.1021/acs.jpcc.9b08500

\section{Published in:}

The Journal of Physical Chemistry C

\section{Citing this paper}

Please note that where the full-text provided on Manchester Research Explorer is the Author Accepted Manuscript or Proof version this may differ from the final Published version. If citing, it is advised that you check and use the publisher's definitive version.

\section{General rights}

Copyright and moral rights for the publications made accessible in the Research Explorer are retained by the authors and/or other copyright owners and it is a condition of accessing publications that users recognise and abide by the legal requirements associated with these rights.

\section{Takedown policy}

If you believe that this document breaches copyright please refer to the University of Manchester's Takedown Procedures [http://man.ac.uk/04Y6Bo] or contact uml.scholarlycommunications@manchester.ac.uk providing relevant details, so we can investigate your claim.

\section{OPEN ACCESS}




\title{
C: Surfaces, Interfaces, Porous Materials, and Catalysis
}

\section{Exploring Voltage Mediated Delamination of Suspended 2D Materials as a Cause of Commonly Observed Breakdown}

\author{
Joshua Loessberg-Zahl, Douwe S. de Bruijn, Wesley T. E. van den Beld, \\ Edwin Dollekamp, Eldad Grady, Ashok Keerthi, Johan G. Bomer, Radha Boya, \\ Harold J.W. Zandvliet, Ageeth A. Bol, Albert van den Berg, and Jan C. T. Eijkel
}

J. Phys. Chem. C, Just Accepted Manuscript • DOI: 10.1021/acs.jpcc.9b08500 • Publication Date (Web): 05 Dec 2019

Downloaded from pubs.acs.org on December 6, 2019

\section{Just Accepted}

"Just Accepted" manuscripts have been peer-reviewed and accepted for publication. They are posted online prior to technical editing, formatting for publication and author proofing. The American Chemical Society provides "Just Accepted" as a service to the research community to expedite the dissemination of scientific material as soon as possible after acceptance. "Just Accepted" manuscripts appear in full in PDF format accompanied by an HTML abstract. "Just Accepted" manuscripts have been fully peer reviewed, but should not be considered the official version of record. They are citable by the Digital Object Identifier (DOI®). "Just Accepted" is an optional service offered to authors. Therefore, the "Just Accepted" Web site may not include all articles that will be published in the journal. After a manuscript is technically edited and formatted, it will be removed from the "Just Accepted" Web site and published as an ASAP article. Note that technical editing may introduce minor changes to the manuscript text and/or graphics which could affect content, and all legal disclaimers and ethical guidelines that apply to the journal pertain. ACS cannot be held responsible for errors or consequences arising from the use of information contained in these "Just Accepted" manuscripts. 


\title{
Exploring Voltage Mediated Delamination of Suspended 2D Materials as a Cause of Commonly Observed Breakdown
}

\author{
J. Loessberg-Zahl ${ }^{\mathrm{a}}$, D.S. de Bruijn ${ }^{\mathrm{a}}$, W.T.E. van den Beld ${ }^{\mathrm{a}, \mathrm{b}}$, E. Dollekamp ${ }^{\mathrm{c}}, \mathrm{E}$. Grady ${ }^{\mathrm{d}}$,A. Keerthie,f, \\ J. Bomer ${ }^{\mathrm{a}}$, B. Radha ${ }^{\mathrm{g}, \mathrm{f}}$, H.J.W. Zandvliet ${ }^{\mathrm{c}}$, A. A. Bol' ${ }^{\mathrm{d}}$ A. van den Berg ${ }^{\mathrm{a}}$, J.C.T. Eijkel ${ }^{\mathrm{a}}$ \\ a BIOS Lab-on-a-Chip Group, University of Twente, MESA+ Institute for Nanotechnology The Netherlands \\ ${ }^{b}$ Industrial Focus Group XUV Optics, University of Twente, MESA+ Institute for Nanotechnology The Netherlands \\ ${ }^{c}$ Physics of Interfaces and Nanomaterials, University of Twente, MESA+ Institute for Nanotechnology The Netherlands \\ d Plasma and Materials Processing, Eindhoven University of Technology, The Netherlands \\ ${ }^{e}$ Department of Chemistry, University of Manchester, United Kingdom \\ ${ }_{\mathrm{f}}^{\mathrm{N}}$ National Graphene Institute, University of Manchester, United Kingdom \\ g Department of Physics and Astronomy, University of Manchester, United Kingdom
}

Abstract

2D barrier materials such as graphene, boron nitride and molybdenum disulfide hold great promise for important applications such as DNA sequencing, desalination, and biomolecular sensing. The 2D materials commonly span pores through an insulating membrane and electrical fields are applied to drive cross-barrier transport of charged solvated species. While the low voltage transmembrane transport is well understood and controllable, high voltage phenomena are uncontrolled and result in the apparent breakdown of the 2D material's critical insulating properties. Here we use suspended graphene over a 50 $\mathrm{nm}$ silicon nitride nanopore as a model system and show that delamination of the 2D-material occurs at higher voltages and can directly cause a number of the puzzling high voltage transport observations. We confirm the occurrence of delamination and observe via AFM measurement a micron scale delaminated patch in a system using CVD graphene. Furthermore, we show that the conductivity of the same system is strongly correlated to the area of delamination via coincident current measurements and optical imaging of the delaminated area. Finally, we demonstrate that delamination alone can cause a dramatic breakdown of barrier function through observation of a reversible increase in conductance of samples prepared with pristine defect-free graphene. These findings should have a great impact on the design and interpretation of 2D-barrier material for both experiments and applications.

\section{Introduction}

2D materials have drawn much attention due to their exceptional mechanical, electrical and transport barrier properties. ${ }^{1,2,3,4,5,6,7,8}$ Envisioned applications of such 2D material membranes abound, for electronic, mechanical or electromechanical devices, or as ultrathin barrier material for biomolecular sensing, desalination or DNA sequencing. 9,10,11,12,13,14,15,16 When used as a barrier material in aqueous systems, their atomic thickness (sub-nm) provides a large advantage over other systems, as pores through 
the material exhibit exceptionally low transport resistance, while the materials themselves are generally impermeable. For such applications, native or manufactured pores of atomic $(\sim \mathrm{nm})$ scale in the barrier material are used. To create a functional barrier device, the $2 \mathrm{D}$ material is generally deposited on a carrier membrane made from silicon nitride $(\mathrm{SiN})$ or silicon oxide $\left(\mathrm{SiO}_{2}\right)$ with larger diameter $(50 \mathrm{~nm}-5$ $\mu \mathrm{m})$ pores. In many cases, the device is then submerged in an aqueous solution and electrical fields are applied over this structure to drive ionic transport.

It has often been observed that applied voltages above a certain threshold (generally 200 to 500 $\mathrm{mV}$ ) result in a breakdown of the resistance of the system, as indicated by a strongly non-linear and sometimes chaotic transmembrane current. $8,9,17,18,19,20$ This apparently consistent observation has been attributed to different phenomena in several studies, being labeled as non-understood ${ }^{19}$, physical damage to the $2 \mathrm{D}$ material ${ }^{9}$ delamination of the $2 \mathrm{D}$ material from the substrate ${ }^{17}$, transient changes in the protonation of charged groups at the pore opening ${ }^{18}$, or evidence for Coulomb blockade ${ }^{20}$. As it is often unclear whether the culprit phenomenon permanently compromises the function of these systems, a proper understanding is of crucial importance.

We explore voltage-mediated delamination of the 2D material from the supporting membrane as the cause for some observations of aberrantly high current at high voltage. The intrusion of a layer of electrolyte between the 2D-material and membrane would provide an alternative conductive pathway through the system, and thus destroy the barrier function and cause the nonlinear current-voltage relation seen at higher voltages. Furthermore, this pathway would be intrinsically unstable, causing chaotic system behavior.

Voltage-mediated delamination has been observed with 2D materials in other scenarios, such as the liquid-phase electrochemical exfoliation of bulk 2D materials and the electrochemical release of 2D materials from their substrate $21,22,23,24$. In these processes, a voltage applied to a bulk 2D material or the substrate on which a 2D material rests causes intrusion of the electrolyte around the 2D material, thus causing delamination. We hypothesized that a similar process is occurring for suspended 2D materials once the transmembrane voltage overcomes a threshold(Figure 1). In this case, the electrolyte solution will begin to encroach into the interface between 2D material and substrate if the resulting decrease in electrical capacitive energy on the 2D material outweighs the cost in energy for wetting the interface. Similarly when the voltage is removed, wetting becomes energetically unfavorable and the 2D material is expected to re-laminate, making the process reversible. This type of delamination would represent an inherent instability in any such suspended configuration in which a transmembrane voltage is applied. 


\section{Results and Discussion}

With an order of magnitude calculation, we can estimate the voltage regime in which delamination is expected. As the process should be spontaneous when the capacitive charging energy is larger than the interfacial energy change $\Delta \Gamma$, we can express the regime via the following inequality,

$$
\frac{V^{2} C_{b}}{2}>\Delta \Gamma
$$

Here $\mathrm{V}$ is the voltage between the two bulk electrolytes on either side of the $2 \mathrm{D}$ material and $\mathrm{C}_{\mathrm{b}}$ is the combined specific capacitance of the 2D-material and the electric double layers that form on the 2D material.

Common 2D materials such as graphene, $\mathrm{MoS}_{2}$ and $\mathrm{BN}$ have comparable reported values for the properties appearing in equation 1 . Specifically, dielectric constants ${ }^{25,26,27,28,43}$ thicknesses $^{26,29,30,31}$ and adhesion energies to both water and ceramic substrates $32,33,34,35,36,37,38,39,40,41$ lie within the same order of magnitude respectively. The most broadly and consistently reported parameters are found for graphenebased systems. Therefore, for the remainder of the theoretical and experimental work presented here, we will work with graphene on SiN systems as a proxy for suspended monolayers of 2D materials in general. While this is not a perfect proxy it should serve to indicate the general range of conditions under which delamination should occur for suspended 2D materials in general.

We will first calculate the expected voltage of delamination to determine if it falls within the typical -1 to $1 \mathrm{~V}$ window where a breakdown is observed. As the $\mathrm{SiN}$ surface has been strongly oxidized in our case (see methods), we use surface energies associated with $\mathrm{SiO}_{2}$. Reliable estimates for the hydroxylated $\mathrm{SiO}_{2}$-water interfacial energy $\left(129 \mathrm{~mJ} / \mathrm{m}^{2}\right)$ and for the graphene-water interface $\left(29 \mathrm{~mJ} / \mathrm{m}^{2}\right)$ are available. ${ }^{36,37}$ Literature values for the interfacial energy of the SiO2/graphene system, on the other hand, vary widely ranging from 450 to $96 \mathrm{~mJ} / \mathrm{m}^{2}$, and are thought to depend on the roughness of the carrier membrane as well as the presence of intermediate water. ${ }^{38,39,40,41}$. We will take the value of approximately $200 \mathrm{~mJ} / \mathrm{m}^{2}$ for the graphene- $\mathrm{SiO}_{2}$ energy in the presence of $\mathrm{H}_{2} \mathrm{O}^{38}$. By subtracting the $\mathrm{SiO}_{2}$-water and graphene-water interfacial energies from the graphene- $\mathrm{SiO}_{2}$ interfacial energy an estimated interfacial energy change $\Delta \Gamma$ of $42 \mathrm{~mJ} / \mathrm{m}^{2}$ is obtained. The capacitor system that is charged consists of the graphene and the two electric double-layers that form on it when a voltage is applied. Specifically, the system is comprised of: the anodic double-layer capacitance (DLC), the graphene capacitance and the cathodic DLC in series. Taking respective values of $0.2,0.08$ and $0.08 \mathrm{~F} / \mathrm{m}^{2} 42,43$, we 
estimate the series capacitance as $33 \mathrm{mF} / \mathrm{m}^{2}$. From these values for interfacial energy and capacitance, we expect a delamination voltage of about $1.6 \mathrm{~V}$. Though approximate, these calculations show delamination may occur near the voltages at which breakdown of these systems is commonly observed.

In the experimental part of this work (Figure 1a), we consider the breakdown of barrier function for both pristine exfoliated graphene and for CVD graphene laden with nanopore defects. While both are expected to delaminate, the behavior of the system after delamination is expected to be slightly different and bears consideration. In both cases, once the voltage threshold for delamination is surpassed, the 2D material is expected to begin delaminating in the area directly around the pore through the substrate material. In the case of CVD graphene, (Figure $1 \mathrm{~b}$ and $\mathrm{c}$ ) as the delaminated patch grows the number of exposed defects increases and the observed electrical resistance of the system should decrease. As a result, the voltage under the 2D material is also expected to decrease with an increased delaminated area, eventually dropping below the voltage threshold for delamination. This would cause the delaminated area to stop growing, once it reaches a specific size. A simple resistive model of this effect can be found in the supplementary information. In the case of the pristine material, (Figure $1 \mathrm{~d}$ and e) there is no decrease in resistance until the delaminated area finds the edge of the $2 \mathrm{D}$ material. The delaminated patch is therefore expected to continue widening until it finds an edge. At this point, current can begin to flow and the voltage under the patch will begin to reduce. From this understanding, the voltage at the observed breakdown is expected to be the same for both CVD and pristine graphene, but the magnitude of current and area of delamination are expected to differ.

To experimentally probe the barrier function breakdown, we have fabricated suspended graphene (either CVD or pristine graphene) on SiN devices. Figure 1a schematically shows the device employed, where a graphene sheet is suspended over a $\mathrm{SiN}$ membrane with a $50 \mathrm{~nm} \varnothing$ nanopore, located between two reservoirs of aqueous salt solutions $(1 \mathrm{M} \mathrm{KCl})$, which are both equipped with a calomel electrode or silver/silver chloride electrode for voltage control. It is worth noting that, as opposed to typical graphene transfer protocols, oxygen plasma treatment of the SiN substrate was performed before transfer of the graphene to the SiN to render the SiN hydrophilic and aid in filling of the SiN nanopore with electrolyte.

First, we validate the occurrence of breakdown in our system via cyclic voltammetry and indeed observe a non-linear current-voltage relationship after a voltage threshold is crossed as previously observed in similar systems by: Jain et al. ${ }^{18}$, Liu et al. ${ }^{9}$, Feng et al. ${ }^{10}$ and Cun et al. ${ }^{8}$. Results for CVD graphene in Figure 2 show the strongly non-linear increase of the current when the voltage exceeds a threshold value of about $0.25 \mathrm{~V}$. Subsequent scans ('cycle 1, 2, 3') show roughly similar responses. It is worth noting that this threshold voltage is lower than predicted, implying either a higher capacitance or a lower adhesion strength. 


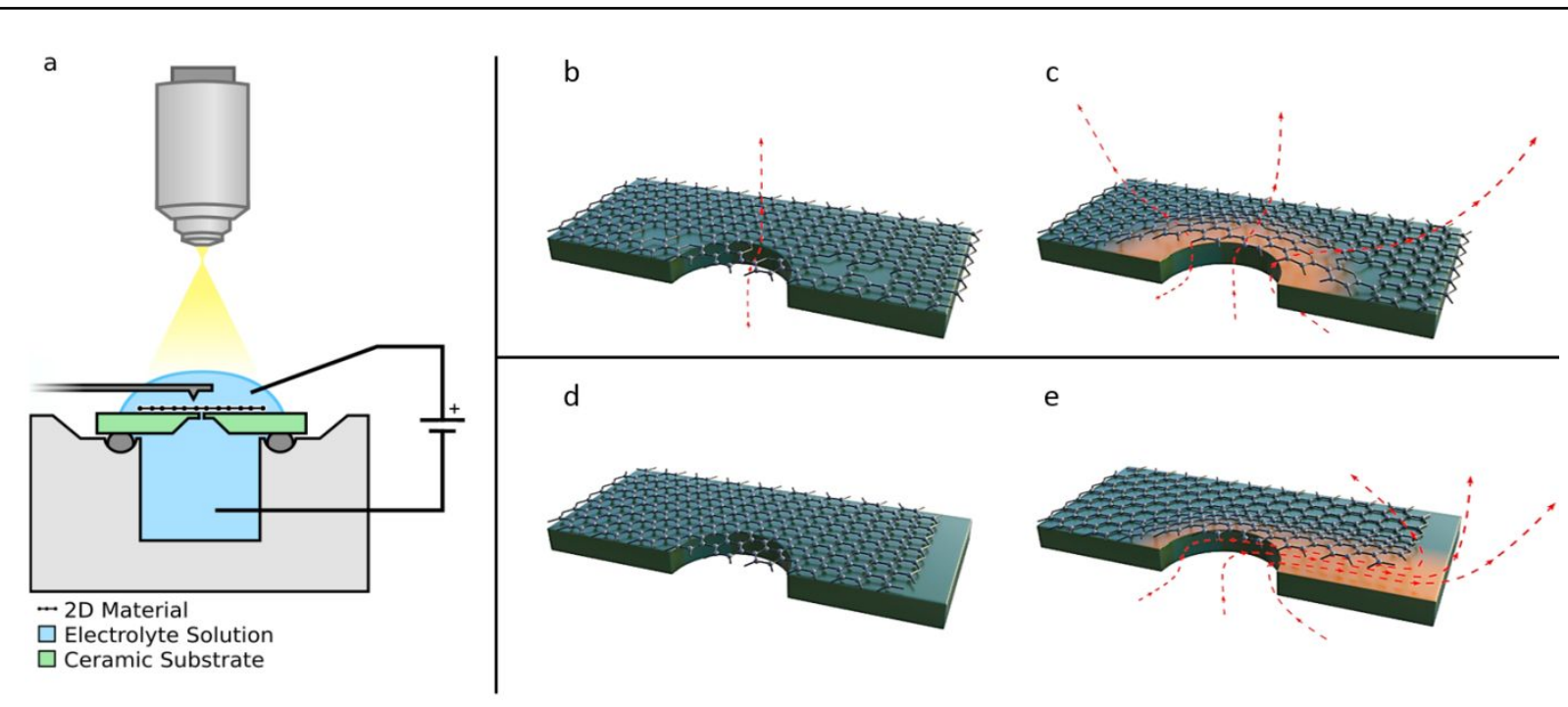

Figure 1: Schematic of a device in the test setup (a). The devices consists of a Si chip with a SiN carrier membrane (green) with graphene (CVD or pristine) overlaid (dotted line). The test setup allows application of voltage and amperometric measurement with coincident optical or AFM measurement. Panels b-e show schematically the system in all the states of the system examined in this paper: CVD graphene before delamination (b) CVD graphene after delamination(c), pristine graphene before delamination (d) and pristine graphene after delamination (e). Orange shaded areas denote the area of delamination and red lines indicate the path of current through the system.

To determine if delamination is occurring concurrently, the graphene was visualized in-situ by inliquid AFM at a series of fixed applied potentials in a similar range to that used during the cyclic voltammetry (Figure 3). A raised patch of graphene above the SiN pore can clearly be seen to appear and disappear with voltage, while the surrounding pre-existing wrinkles in the CVD graphene layer remain relatively unchanged. The degree of lifting appears to depend on the voltage applied as seen by: Figure 3 $\mathrm{a}-\mathrm{b}$ showing the difference between $0 \mathrm{~V}$ and $0.5 \mathrm{~V}$, Figure $3 \mathrm{c}-\mathrm{d}$ showing the difference between $0 \mathrm{~V}$ and $1.0 \mathrm{~V}$, and Figure $3 \mathrm{e}-\mathrm{f}$ showing the difference between $0 \mathrm{~V}$ and $1.5 \mathrm{~V}$. This clearly indicates that the application of voltage is causing the graphene to lift from the surface.

We also note from the AFM measurements that the delamination of the CVD graphene remains localized in our system. As mentioned before, we expect this because, as delamination progresses, the current through the system may pass through an increasing number of nanopore defects reducing the voltage under the graphene. With this understanding of the system, it becomes clear that the measured system conductance for CVD graphene is expected to be highly correlated with the observed delaminated area. To expand upon this, some basic modeling of the expected conductance and correlation to the delaminated area is included in the supplementary information. 
We empirically determine the correlation between delaminated area and system conductance via coincident optical reflection microscopy and cyclic voltammetry. The delaminated area was calculated from the optical data. Figure 4 a-e show this data for one device while Figure $4 \mathrm{f}-\mathrm{j}$ show it for another. In particular, Figures $4 \mathrm{e}$ and $4 \mathrm{j}$ show the measured area plotted against the conductance. A strong correlation is observed in both cases, providing strong evidence that delamination is contributing to the observed nonlinear conductance.

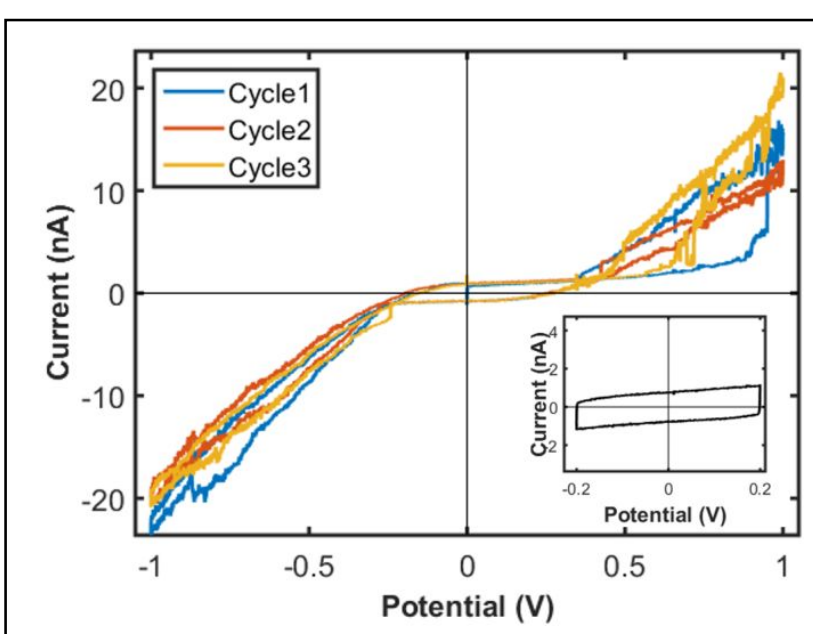

Figure 2: Sample cyclic voltammogram from the CVD graphene on $\mathrm{SiN}$ system. The dramatic increase in current at about $\pm 0.25 \mathrm{~V}$ constitutes the discussed breakdown of barrier function. Note that the current not only increases more steeply at higher voltages but also becomes somewhat chaotic, sometimes changing dramaticly for a small change in voltage. The inset shows the often reported current response for a low voltage sweep which shows no breakdown. It is worth noting that cyclic voltammetry results in a non-zero capacitive charging current at zero volts compared to the sequential fixed voltage measurements often used in the literature. This current is directly proportional to the scan rate and goes to zero for fixed voltage application.
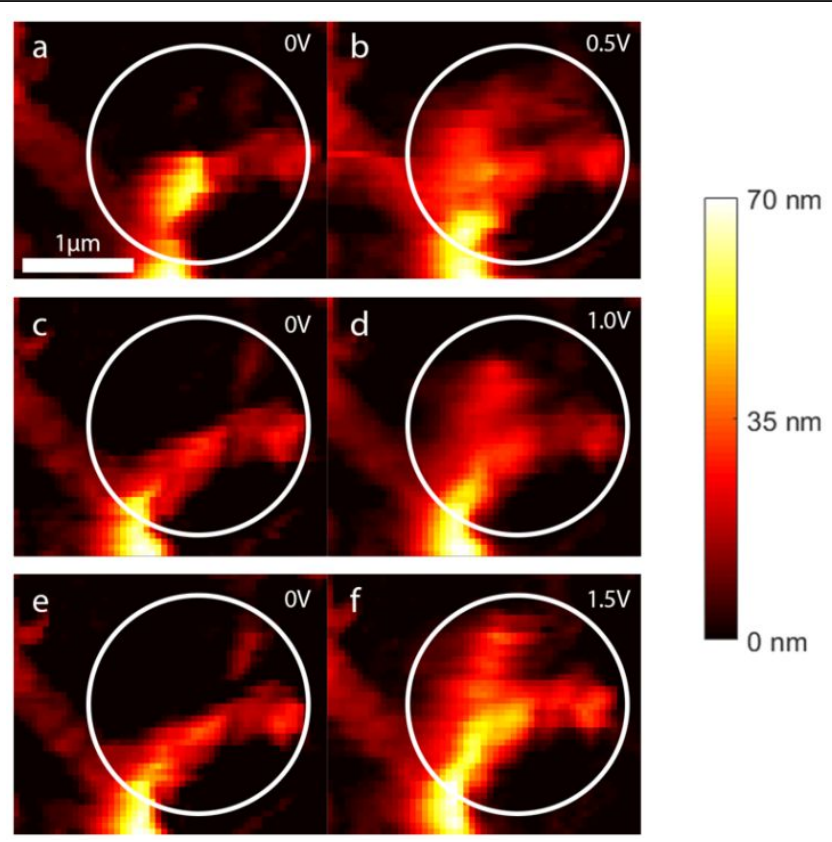

Figure 3: Sequential AFM measurements of changes in CVD graphene surface profile during various applied voltages. Voltages of $0.5(\mathrm{~b}), 1.0(\mathrm{~d})$, and $1.5(\mathrm{f})$, V were used. After application of a given voltage a subsequent scan is performed at $0 \mathrm{~V}(\mathrm{a}, \mathrm{c}, \mathrm{e})$ shown in the left column. A raised patch can be seen to appear and disappear with the application and removal of voltage implying that delamination is occurring. White circle indicates the area in which the SiN nanopore lies as validated by SEM imaging (Figure S6). Note that the elevated features during application of zero volts are preexisting wrinkle in the graphene.

As also observed by the AFM measurements, the local delamination in our system is strongly influenced by the local graphene topology, such as wrinkles, which may account for the heterogeneity within cycles and between different systems. The continuous and unpredictable formation of unstable, but sometimes permanent pathways can explain both the chaotic behavior and the permanent delamination 
observed. The discrete nature of defects in the CVD graphene may also contribute to the chaotic and step-like changes in current. This idea is further expanded upon in the supplementary information.

While the above explains why an increased delaminated area is often concurrent with an increased conductivity, it does not prove conclusively that delamination on its own can cause the observed breakdown of barrier function. Indeed we sometimes observed an elevated conductance without a direct increase in the delaminated area. Likewise, an increase in area is not always immediately followed by an elevated conductance (for example: Figure 4i, 500-600 seconds). Other possible phenomena such as nonlinear transport through defects in the graphene or physical damage to the graphene may therefore also be at play. $.18,19,20$
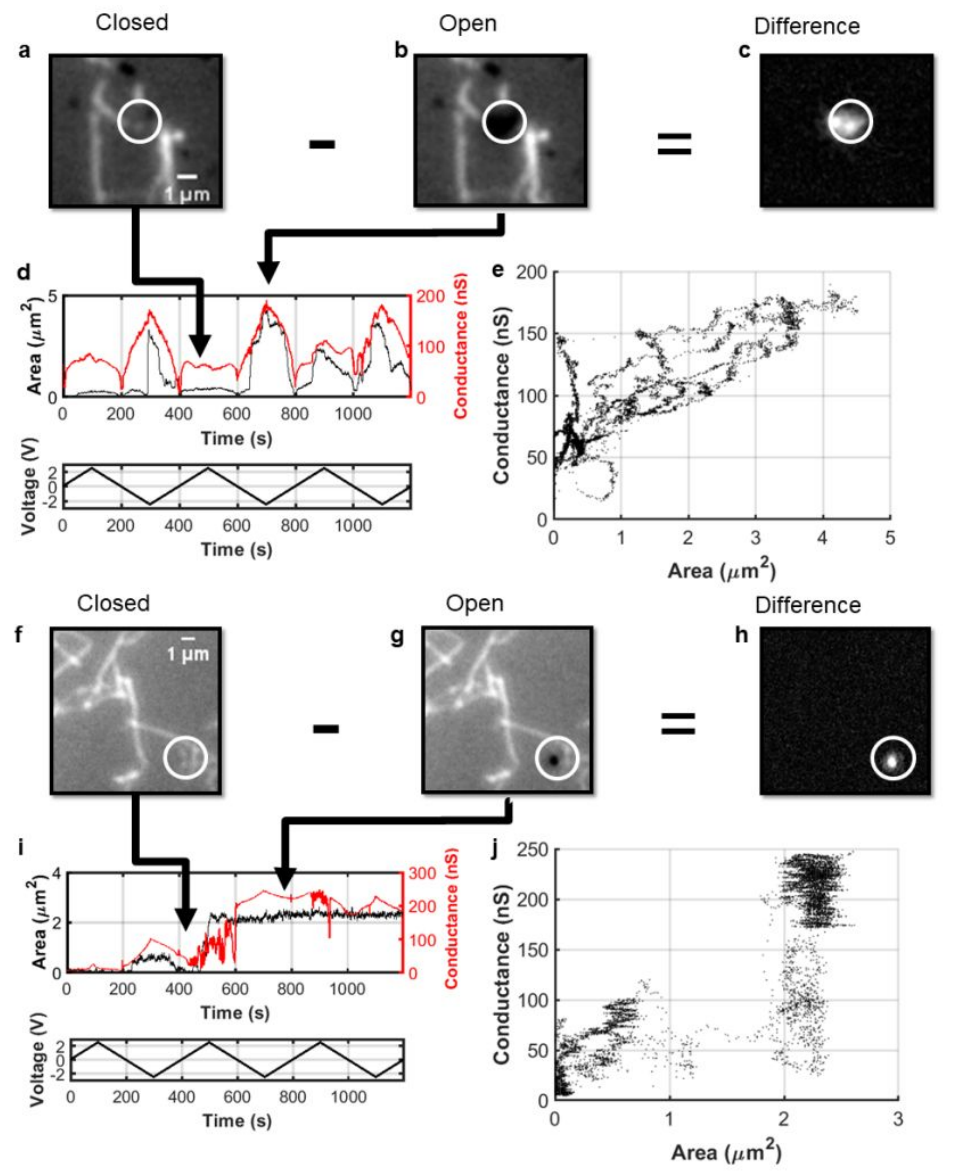

Figure 4: Optically determined area of delamination and coincident conductance of CVD graphene for two devices (a-e and $\mathrm{f}-\mathrm{j})$ plotted both against time $(\mathrm{d}, \mathrm{i})$ and against each other $(\mathrm{e}, \mathrm{j})$. The strong correlation shows that delamination often coincides with barrier breakdown and likely contributes to it. Sample optical data from region of delamination is also shown (a-c, f-h) and differential images demonstrate that the effect is local as expected (c,h). White circle indicates the area in which the SiN nanopore lies as validated by SEM imaging (Figure S6). The appearance of a dark spot indicates the formation of a blister of liquid under the graphene. Area of delamination is approximated by the number of pixels above a threshold value after normalization and background subtraction. Top data set shows a device where the graphene appears to delaminate at high voltage and relaminates at low voltage. Bottom dataset shows a device where the graphene remains delaminated after the initial delamination event and the conductance similarly remains high. Note that the white lines appearing on the optical images are preexisting wrinkles in thepgrapheng lus Environment 
To test if delamination causes a breakdown in the absence of other possible causes, devices were prepared with defect-free exfoliated graphene and tested similarly with cyclic voltammetry (Figure 5). In this case, there are no defects so any observed breakdown in barrier function necessarily comes from either the delamination of the graphene or the permanent physical degradation of the graphene layer.

In these experiments, we indeed see a significant breakdown in barrier function at sufficiently high applied voltages as well as a total recovery of barrier function after returning to lower voltages. While physical degradation of the graphene may seem to be a likely explanation for the initial breakdown, it would result in a permanently increased conductance of the system, which was not observed. However, in the case of delamination-mediated breakdown, the increased conductance must be caused by the reversible opening of a conductive path under the graphene sheet from the ceramic pore to the edge of the graphene flake (Figure 1e). We note that the resulting path must be much longer (Figure S5) than the path under the graphene observed in the case of

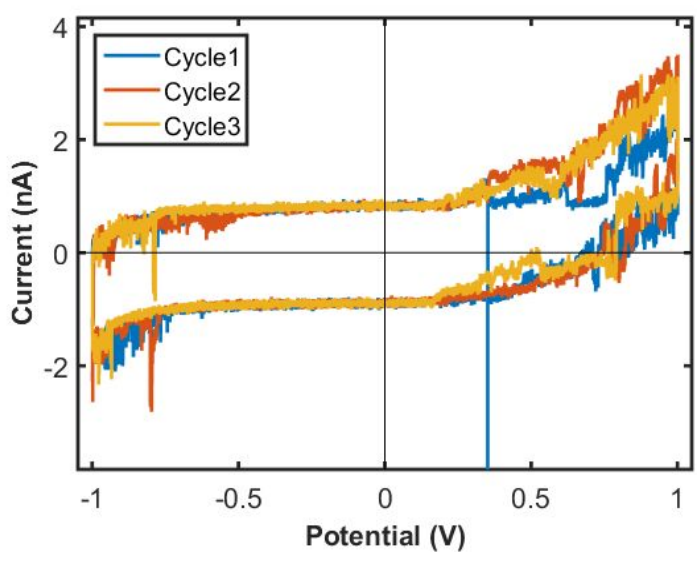

Figure 5: Three cyclic voltammograms of the pristine graphene-on-SiN system. Breakdown is again indicated by a nonlinear increase in the current at about 0.25 volts and again the current not only increases more steeply but also becomes chaotic. In contrast to the CVD graphene there are no nanopore defects to contribute to the current and the reversibility of the breakdown implies that there is no physical damage to the graphene itself. Therefore delamination remains the single explanation for the observed breakdown under these conditions.

CVD graphene and thus the increase in conductivity is expected to be less dramatic.(See SI for expanded explanation) Removal of voltage will allow this path to collapse and cause the conductance to recover to its initial level, matching our observations. Therefore we take the reversible breakdown of barrier function in the case of pristine graphene as confirmation that delamination can be solely responsible for the breakdown in suspended 2D material systems.

To help diagnose delamination in future experiments we include below descriptions of three signature features of the voltammogram produced during delamination. We also include the alternative explanation for the behavior that each signature rules out. First, the process must be reversible, meaning that the same low voltage conductivity is recovered at the end of each cycle. If the nonlinear current were caused by the generation of defects in the 2D material or the SiN membrane, the current observed at low voltage is expected to increase dramatically from cycle to cycle as more conductive paths are opened. Second, delamination causes step-like changes and an increased noise in the high voltage current. This is 
due to the discrete nature of the defects exposed by delamination. In the case of nonlinear voltammograms caused by asymmetric charge on a nanopore or coulomb blockade, the conductivity is expected to increase smoothly with voltage. Finally, the low voltage capacitance and current noise are comparable to that of a system without any 2D material. If a bubble in the $\mathrm{SiN}$ nanopore were interfering with the measurement, capacitance or current noise would be elevated at low voltage.

\section{Conclusion}

We have investigated voltage-mediated delamination of suspended 2D materials via a graphene on SiN system and observed the effect of delamination on the breakdown of barrier function. For both exfoliated and CVD graphene, we observe breakdown of the system reliably at $\sim 200 \mathrm{mV}$. Via in-liquid AFM, we confirmed that a micron-scale raised blister in the CVD graphene does indeed occur above the $50 \mathrm{~nm}$ SiN pore at above the breakdown voltage. Next, we found that the degree of delamination of the CVD graphene is strongly correlated with the conductance of the system during breakdown, which indicates with a strong likelihood that delamination contributes to the breakdown. We then demonstrated that delamination can be the sole cause of breakdown by repeatedly inducing and reversing breakdown of a system made with mechanically exfoliated pristine graphene. These results show that voltage-mediated delamination needs to be accounted for when designing systems that utilize suspended 2D materials. If the barrier function of the $2 \mathrm{D}$ material is critical, steps need to be taken to enhance adhesion of the $2 \mathrm{D}$ material, especially when higher transmembrane voltages are needed.

\section{Conflicts of Interest}

There are no conflicts of interest to declare.

\section{Supporting Information:}

- Description of the setup

- Simple model of the system

- Discussion of chaotic dynamics

- Discussion of the current under the exfoliated graphene

- Discussion of Raman spectra of the used graphene

- 2 videos of the process and coincident conductivity.

\section{Acknowledgements:}


We would like to thank Vasilis Papadimitriou for much fruitful discussion and help with student supervision. Henk van Wolferen also was of great assistance for much of the focused ion beam milling. This work was supported in part by the Netherlands Center for Multiscale Catalytic Energy Conversion (MCEC), an NWO Gravitation program funded by the Ministry of Education, Culture, and Science of the government of the Netherlands. B. Radha acknowledges Royal society Fellowship R123098 and RGF\EA $\backslash 181000$ for contribution to this work. E. Grady acknowledges the financial support of the Dutch Technology Foundation STW (project number 140930), which is part of the Netherlands Organization for Scientific Research (NOW). A. Keerthi thanks Ramsay Memorial Fellowships Trust for the award.

\section{References:}

\footnotetext{
${ }^{1}$ Hamm, J.M.; Hess, O.; Two Two-Dimensional Materials Are Better than One, Science, 2013, 340, 1298-1299.

2 Novoselov, K. S.; Jiang, D.; Schedin, F.; Booth, T. J.; Khotkevich, V. V.; Morozov, S. V.; Geim, A. K. Two-Dimensional Atomic Crystals, Proc. Natl Acad. Sci. USA, 2005, 102, 10451-10453

${ }^{3}$ Novoselov, K. S.; Geim, A.K.; Morozov, S. V.; Jiang, D.; Zhang, Y.; Dubonos, S.V.; Grigorieva, I.V.; Firsov, A.A. Electric Field Effect in Atomically Thin Carbon Films, Science, 2004, 306, 666-669.

${ }^{4}$ Butler, S. Z.; Hollen, S.M.; Cao, L.; Cui Y.; Gupta, J.A.; Gutierrez, H.R.; Heinz, T.F.; Hong, S.S.; Huang, J.; Ismach, A.F.; et.al; Progress, Challenges, and Opportunities in Two-Dimensional Materials Beyond Graphene, ACS Nano, 2013, 7, 2898-2926,

${ }^{5}$ Guo, S.; Dong, S.; Graphene Nanosheet: Synthesis, Molecular Engineering, Thin Film, Hybrids, and Energy and Analytical Applications, Chem. Soc. Rev. 2011, 40, 2644-2672.

${ }^{6}$ Fiori, G.; Bonaccorso, F.; lannaccone, G.; Palacios, T.; Neumaier, D.; Seabaugh, A.; Banerjee, S.K.; Colombo, L. Electronics Based on Two-Dimensional Materials, Nature Nanotech., 2014, 9, 768-779.

7 Liu, S.; Lu, B.; Zhao, Q; Li, J.; Gao, T.; Chen, Y.; Zhang, Y.; Liu, Z; Fan, Z.; Yang, F.; et al., Boron Nitride Nanopores: Highly Sensitive DNA Single-Molecule Detectors, Adv. Mater., 2013, 25, 4549-4554.

${ }^{8}$ Cun, H.; Hemmi, A; Miniussi, E.; Bernard, C.; Probst, B.; Liu, K.; Alexander D.T.L.; Kleibert, A.; Mette G.; Weinl M.; et al., Centimeter-Sized Single-Orientation Monolayer Hexagonal Boron Nitride With or Without Nanovoids, Nano Lett. 2018, 18, 1205-1212.

${ }^{9}$ Liu, K.; Feng, J.; Kis, A.; Radenovic, A. Atomically Thin Molybdenum Disulfide Nanopores with High Sensitivity for DNA Translocation, ACS Nano, 2014, 8, 2504-2510.

${ }^{10}$ Feng, J.; Liu, K.; Bulushev, R.D.; Khlybov, S.; Dumcenco, D.; Kis, A.; Radenovic, A. Identification of Single Nucleotides in MoS2 Nanopores, Nature Nanotech. 2015, 10, 1070-1076.

${ }^{11}$ Garaj, S.; Hubbard, W.; Reina, A.; Kong, J.; Branton D.; Golovchenko, J.A.; Graphene as a Subnanometre TransElectrode Membrane. Nature, 2010, 467, 190-193.

12 Merchant, C.A.; Healy, K.; Wanunu, M.; Ray, V.; Peterman, N.; Bartel, J.; Fischbein, M.D.; Venta, K.; Luo, Z.; Johnson, A.T.C.; et.al., DNA Translocation Through Graphene Nanopores, Nano Lett. 2010, 10, 2915-2921.

${ }^{13}$ Schneider, G.F.; Kowalczyk, S.W.; Calado, V.E.; Pandraud, G.; Zandbergen, H.W.; Vandersypen, L.M.K.; Dekker, C. DNA Translocation through Graphene Nanopores, Nano Lett. 2010, 10, 3163-3167.

${ }^{14}$ Heerman, S.; Dekkar, C. Graphene Nanodevices for DNA Sequencing. Nature Nanotech. 2016, 11, 127-136.

${ }^{15}$ Venkatesan, B.; Bashir; R. Nanopore Sensors for Nucleic acid Analysis. Nature Nanotech . 2011, 6, 115-624.

${ }^{16}$ Wu, Y.; Tilley, R.; Gooding, J. Challenges and Solutions in Developing Ultrasensitive Biosensors, Journal of the American Chemical Society, 2019, 141, 1162-1170.

${ }^{17}$ Esfandiar, A.; Radha, B.; Wang, F.C.; Yang, Q.; Hu, S.; Garaj, S.; Nair, R.R.; Geim, A.K.; Goopinadhan, K.; Size Effect in Ion Transport Through Angstrom-Scale Slits, Science, 2017, 358, 511-513.

18 Jain, T.; Rasera, B.C.; Guerrero, R.J.S.; Boutilier, M.S.H.; O'Hern, S.C.; Idrobo, J.C.; Karnik, R. Heterogeneous SubContinuum Ionic Transport in Statistically Isolated Graphene Nanopores, Nature Nanotech. 2015, 10, 1053.
} 
${ }^{19}$ Surwade, S.P.; Smirnov, S.N.; Vlassiouk, I.V.; Unocic, R.R.; Veith, G.M. Dai, S.; Mahurin, S.M. Water Desalination Using Nanoporous Single-Layer Graphene, Nature Nanotech., 2015, 459-464.

${ }^{20}$ Feng, J.; Liu, K.; Graf, M.; Dumcenco, D.; Kis, A.; Di Ventra M.; Radenovic, A.; Observation of Ionic Coulomb Blockade in Nanopores, Nature Materials, 2016, 15, 850-856.

${ }^{21}$ Zhang, J.; Wang, E. STM Investigation of HOPG Superperiodic Features Caused by Electrochemical Pretreatment, J. Electroanal. Chem. 1995, 399, 83-89.

22 Alliata, D.; Häring, P.; Haas, O.; Kötz, R.; Siegenthaler, H.; Anion Intercalation Into Highly Oriented Pyrolytic Graphite Studied by Electrochemical Atomic Force Microscopy, Electrochem. Comm. 1999, 1, 5-9.

${ }^{23}$ Verguts, K.; Coroa, J.; Huyghebaert, C.; De Gendt, S.; Brems. S. Graphene Delamination Using 'Electrochemical Methods': an Ion Intercalation Effect. Nanoscale. 2018, 10, 5515-5521.

${ }^{24}$ Wang, Y.; Zheng, Y.; Xu, X.; Dubuisson, E.; Bao, Q.; Lu, J.; Loh. K. Electrochemical Delamination of CVD Grown Graphene Film: Towards Recyclable use of Copper Catalyst. ACS Nano. 2011, 5, 9927-9933.

${ }^{25}$ Dean, C.; Young, A.; Meric, I.; lee, C.; Wang, L.; Sorgenfrei, S.; Watanbe, K.; Taniguci, T.; Kim, P.; Shepard, K.; et.al; Boron Nitride Substrates for High-Quality Graphene Electronics. Nature Nanotech, 2010, 5, 722-726.

${ }^{26}$ Chen, X,; Wu, Z.; Xu, S.; Wang, L.; Huang, R.; Han, Y.; Ye, W.; Xiong, W.; Han, T.; Long, G.; et.al. Probing Electron States and Electron-Insulator Transition Mechanisms in Molybdenum-Disulfide Vertical Heterostructures. Nature Communications. 2015, 6, 6088.

${ }^{27}$ Cheiwchanchamnangij, T.; Lambrecht. W. Quasiparticle Band Structure Calculation of Monolayer Bilayer and Bunk MoS 2 . Physical Review B. 2005, 85, 302, 2012.

${ }^{28}$ Molina-Sanchez, A.; Wirtz, L.; Phonons in Single Layer and Few Layer $\mathrm{MoS}_{2}$ and WS 2 . Physical Review B. 2011, 84 155413.

${ }^{29}$ Liu, S. ; Lu, B.; Zhao, Q.; Li, J.; Gao, T.; Chen, Y.; Zhang, Y.; Liu, Z.; Fan, Z.; Yang, F.; et. al. Boron Nitride Nanopores: Highly Sensitive DNA Single-Molecule Detectors. Advanced Materials. 2013, 25, 4549-4554.

30 Joo, M.; Moon, B.; Ji, H.; Han, G.; Kim. H.; Lee, G.; Lim, S.; Suh, D.; Lee Y. Electron Excess Doping and Effective Schottkey Barrier Reduction on the $\mathrm{MoS}_{2} / \mathrm{h}-\mathrm{BN}$ Heterostructure. Nano letters. 2016, 16, 6383-6389.

${ }^{31} \mathrm{Ni}$, Z.; Wang, H.; Kasim, J.; Fan, H.; Yu, T.; Wu, Y.; Fang, Y.; Shen, Z. Graphene Thickness Determination Using Reflection and Contrast Spectroscopy. Nano Letters. 2007, 7, 2758-2763.

${ }^{32}$ Lee, C.; Li, Q.; Kalb, W.; Liu, X.; Berger, H.; Carpick, R.; Hone, J. Frictional Characteristics of Atomically Thin Sheets. Science. 2010, 2, 76-80.

${ }^{33}$ Yum, K.; Yu, M. Measurement of Wetting Properties of Individual Boron Nitride Nanotubes with the Wilhelmy Method Using a Nanotube-Based Force Sensor. Nano Letters. 2016, 6, 329-333.

${ }^{34}$ Deng, S.; Gao, E.; Xu, Z.; Berry, V. Adhesion Energy of $\mathrm{Mo}_{2} \mathrm{~S}$ Thin Films on Silicon Based Substrates Determined via the Attributes of a Single $\mathrm{MoS}_{2}$ Wrinkle. Applied Materials and Interfaces. 2017, 9, 7812-7818.

${ }^{35}$ Kozbial, A.; Gong, X.; Liu, H.; Li. L.; Understanding the Intrinsic Water Wettability of Molybdenum Disulfide $\left(\mathrm{MoS}_{2}\right)$. Langmuir. 2015, 31, 8429-8435.

${ }^{36}$ Brunauer, S.; Kantro, D.; Weise, C. The Surface Energies of Amorphous Silica and Hydrous Amorphous Silica. Canadian J. Chem. 1956, 34, 1483-1496.

37 Wang, S.; Zhang, Y.; Abidi, N.; Cabrales L.; Wettability and Surface Free Energy of Graphene Films, Langmuir, 2009, 25, 11078-11081.

${ }^{38}$ Gao, W.; Xiao, P.; Henkelman, G.; Liechti, K. M.; Huang, R. Interfacial Adhesion Between Graphene and Silicon Dioxide by Density Functional Theory with Van Der Waals Corrections. Journal of Physics D: Applied Physics, 2014, 47, 255301.

39 Paek, E.; Hwang, G.S. A Computational Analysis of Graphene Adhesion on Amorphous Silica, Journal of Applied Physics 2013, 113, 164901.

${ }^{40}$ Yue, K.; Gao, W.; Huang, R.; Liechti, K.; Analytical Methods for the Mechanics of Graphene Bubbles, Journal of Applied Physics, 2012, 112, 083512.

${ }^{41}$ Boddeti, N.; Liu, X.; Long, R.; Xiao, J.; Bunch, J.; Dunn, M. Graphene Blisters with Switchable Shapes Controlled By Pressure and Adhesion, Nano Letters. 2013, 13, 6216-6221.

42 Jiang, G.; Cheng, C.; Li, D.; Liu, J.Z Molecular Dynamics Simulations of the Electric Double Layer Capacitance of Graphene Electrodes in Mono-Valent Aqueous Electrolytes, Nano Res. 2016, 9, 174-186. 
1

2

3

4

5

6

7

8

9

10

11

12

13

14

15

16

17

18

19

20

21

22

23

24

25

26

27

28

29

30

31

32

33

34

35

36

37

38

39

40

41

42

43

44

45

46

47

48

49

50

51

52

53

54

55

56

57

58

59

60

${ }^{43}$ Xia, J.; Chen, F.; Li, J.; Tao, N. Measurement of the Quantum Capacitance of Graphene, Nature Nanotech., 2009, 4, 505-509. 


\section{TOC Figure:}

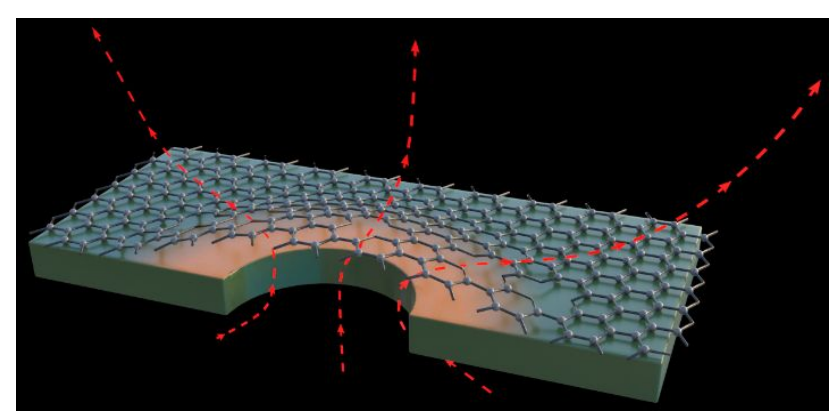

